\section{政 Heighten Science \\ P U B L I C A T I O N S Corporation}

ISSN

2639-3220

\title{
Neurobiology of Common Sleep Disorders
}

\author{
Zhao Liu*, Abdullah Tolaymat and Sreenivas K Avula \\ Department of Pediatrics and Division of Pediatric Neurology, Children's Hospital of Illinois, University \\ of Illinois College of medicine at Peoria, 520 N.E. Glen Oak Ave, Peoria, IL 61637-0001, USA
}

\begin{abstract}
*Address for Correspondence: Zhao Liu, Department of Pediatrics and Division of Pediatric Neurology, Children's Hospital of Illinois, University of Illinois College of Medicine, Peoria, 520 N.E. Glen Oak Ave, Peoria, IL 616370001, USA, Tel: (309) 634 2603; Fax: (309) 624 8884; Email: liuzh@uic.edu
\end{abstract}

Submitted: 28 July 2018

Approved: 13 August 2018

Published: 14 August 2018

Copyright: @ 2018 Liu Z, et al. This is an open access article distributed under the Creative Commons Attribution License, which permits unrestricted use, distribution, and reproduction in any medium, provided the original work is properly cited.

Keywords: Sleep disorder; Insomnia; Hypersomnia; Parasomnia; Circadian Rhythms; Sleep-related movement disorders; Narcolepsy; Circadian rhythm disorder; Drosophilae; Fruit fly; Circadian gene period

\section{Abstract}

Sleep disorders in human are common and detrimental to general health of all age groups. While the neurobiological mechanisms of sleep disorders are not yet fully understood, recent advances in research on the sleep-wake regulation mechanism, genetic and epigenetic factors, cognitive, emotional and physiological changes related to sleep have shed light on the mechanistic basis of sleep disorders. Over the past two decades, studies in Drosophila have yielded new insights into basic questions regarding sleep function and regulation. More recently, powerful genetic approaches in the fly have been applied toward studying primary human sleep disorders and other disorders associated with dysregulated sleep. In this review, we discussed recent advances in neurobiology of sleep-wave cycle and common sleep disorders. Understanding these mechanisms are important in the diagnosis, treatment and prevention of these common disorders.

\section{Introduction}

Sleep is important part of life for the maintenance of biological functions, such as homeostasis, metabolic restoration, physiological regulation, and adaptive cognitive functions in the central nervous system [1]. Sleep disorders are one of the most common disorders affecting the general health in people across all age groups ranging from infant to elderly. Accumulating evidence have shown that sleep disorders are closely related to childhood developmental disorders, cardiovascular disease, pulmonary dysfunctions, neurological disorders and other chronic health problems [2]. Recent work has begun to shed light on the mechanistic basis of sleep disorders such as insomnia, hypersomnia, parasomnia, sleep related movement disorders and circadian rhythm disorders. Over the past 20 years, studies in Drosophila have yielded new insights into basic questions regarding sleep function and regulation. More recently, powerful genetic approaches in the fly have been applied toward studying primary human sleep disorders and other disease states associated with dysregulated sleep. Sleep-wake cycle is a complex physiological process regulated by brain activity, and multiple neurotransmitter system such as monoamines, acetylcholine, excitatory and inhibitory amino acids, peptides, purines, and neuronal and non-neuronal humoral modulators such as cytokines and prostaglandins [3]. It is well established that the sleep-wake cycle is mostly controlled by the ascending reticular activating system including the ventrolateral preoptic nucleus, the median preoptic nuclei, and a group of orexinergic neurons in the lateral hypothalamus. The main neurotransmitters in ascending reticular activating system include cholinergic, monoaminergic, histaminergic, and glutamatergic neurons in the brainstem, especially in the laterodorsal pontine tegmentum and pedunculopontine tegmentum, locus coeruleus, tuberomammilary nucleus, and dorsal raphe. These structures project to the thalamus, basal forebrain, and cerebral cortex, and are crucially involved in the generation of wakefulness [4]. 
The study of sleep in small model organisms has important implications for our understanding of sleep in higher order systems. It has been demonstrated that many of the major neurochemical components and signaling pathways that regulate sleep in Drosophila are conserved in mammalian sleep. New research discoveries continue to confirm that many of the general biological principles dictating how sleep and wake are controlled in the fly also exist in more complex species [5]. The identification of an interconnection between sleep and circadian rhythms has been recapitulated in many mammalian models since the early Drosophila research identifying the circadian genes and proteins that maintain the daily patterns of sleep and wake. It has been shown fruit fly sleep bears resemblance to sleep in higher order organisms, i.e, sleep and wake cycle are partially determined by the activation or inhibition of specific brain circuits. Within these sleep circuits, there is data to suggest that mutually inhibitory connections may be fundamental for state switching; this is true in both the fly and mammals [6]. Drosophila studies have demonstrated that there are multiple neuronal groups involved in sleep regulation that are analogous to mammalian nuclei, and a complete understanding of the Drosophila sleep circuit may further elucidate mammalian sleep circuits [7]. There is also an increasing focus on sleep regulation by extraneuronal cell types that has been moved forward by some work in the fly. Sleep signaling from the fat bodies in Drosophila provided early experimental evidence that peripheral signals can change sleep states and work in mammals confirm not only that peripheral tissues are affected by sleep, but also that peripheral signaling can regulate behavioral state. Therefore, studies in the fruit fly continue to align with the growing body of work that is demonstrating that sleep is not only controlled by the brain but also by the interaction between the brain and the rest of the body. In all species studied to date including Drosophila, it is evident that sleep affects a very wide range of biological processes. This suggests that sleep likely serves multiple functions that are not only specific to the brain but have importance for peripheral organs as well. This notion is not particularly surprising given the pervasiveness of sleep across the animal kingdom and throughout evolution [8].

\section{Neurobiology of insomnia}

Insomnia is the most common sleep complaint. According to Diagnostic and Statistical Manual of Mental Disorders (DSM)-5, insomnia is defined as difficulty falling asleep, difficulty initiating or maintaining sleep, or non-restorative sleep at least 3 times a week for a minimum of 90 days and causing clinically significant distress [9]. It involves both a sleep disturbance and daytime symptoms which can negatively affects overall quality of life. As many as $30 \%$ to $35 \%$ of adults complain of insomnia, especially older adults, women, people under stress and people with certain medical and mental health problems such as depression. While the neurobiological mechanisms of insomnia are not yet fully understood. Recent studies showed that sleep-wake regulation mechanism, genetic and epigenetic factors, cognitive, emotional and physiological hyperarousal are important factors for the development and maintenance of insomnia [10]. It has been postulated that a misalignment of the circadian process can lead to phase delays or advances might lead to either prolonged sleep onset latency or early morning awakening. These changes might also be shown by delayed or advanced melatonin secretion. Dysfunction of the homoeostatic process could explain both sleep onset and sleep maintenance difficulties. The homoeostatic process has been specifically linked to extracellular adenosine concentration in the basal forebrain, which rises as sleep pressure increases. At the neurochemical level, several mechanisms of sleep-wake regulation have been identified. Reduced GABAergic activity or orexinergic overactivity might be associated with a weakening of the sleep-promotion system or a strengthening of the arousal system, respectively.

Feige et al., showed many patients with insomnia had increased frequency of brief events such as shifts in sleep stages between Non-REM and REM sleep. Among Non- 
REM stages, brief periods of awakening and microarousals (brief and transient changes in EEG frequency suggestive of an awake state), and not by extremely long periods of wakefulness [11]. As compared with healthy sleepers, people with insomnia, have the most pronounced differences in the EEG fast frequency range ( $\beta$ power). This type of instability has also been suggested to be relevant for the disruption of REM sleep, which is known to be especially fragmented in insomnia patients, with microarousals. It should be noted, however, that some patients with insomnia do show strong disturbances in sleep continuity, including long sleep latencies or protracted periods of wakefulness occurring during the night. A 2014 meta-analysis of polysomnographic data showed that, on average, the sleep of patients with insomnia is shortened by about 25 min when compared with good sleepers.

Genetics and epigenetics studies in familiar insomnia cases have provided strong evidence for a familial aggregation of insomnia [12]. In afflicted individuals, between $35 \%$ and $55 \%$ of first-degree relatives also have insomnia, which is a substantially higher rate of insomnia than that seen in first-degree relatives of good sleepers. To separate genetic and environmental factors, several twin studies have been done yielding heritability coefficients between $42 \%$ and $57 \%$. Moreover, a twin study suggests that the vulnerability for the development of stress-induced sleep disturbances, as measured by the Ford Insomnia Response to Stress Test (FIRST), is genetically influenced, with heritability estimates of about $29 \%$ for women and $43 \%$ for men [13]. Drosophila is an excellent model system for studying human neurobiology of sleep disorders since both behavioral and electrophysiological features of sleep in Drosophila are reminiscent of mammalian sleep. Many factors controlling sleepwake states identified from genetic approaches in Drosophila have already emerged as having analogous functions in humans. Thirty years after the circadian gene period (PER) was discovered in Drosophila, genetic analyses of humans with familial advanced sleep phase syndrome, delayed sleep phase syndrome, and extreme diurnal preference have revealed various mutations in human PER homologs. In addition, forward genetic screens in flies have implicated potassium channels in the regulation of sleep. Josephs et al have found autoantibodies against potassium channels are associated with the human disease Morvan's syndrome, which often manifests with severe insomnia [14]. Moreover, Cirelli et al., have reported that drugs such as caffeine, amphetamine, and modafinil have similar effects on sleep and wake in flies and humans, providing more evidence for the molecular conservation of sleep regulatory mechanisms.

The search for specific genotypes of insomnia has identified various candidate genes. Mutations have been identified in CLOCK genes genes [15] coding for the $\beta 3$ subunit of the $\mathrm{GABA}_{\mathrm{A}}$ receptor [16] and serotonin transporter genes [17]. However, none of these results have been replicated in independent samples. Barclay et al have recently suggested that epigenetic mechanisms might be involved in the development and maintenance of insomnia [18]. It has been hypothesized that stressful life events could have the capability to change the activity of stress-regulatory systems. This change, in turn, might induce long-term changes in brain structures such as the hippocampus. The hippocampus is an especially plastic brain region vulnerable to stress and a target of stress hormones; furthermore, it has been shown that hippocampal neurogenesis, and in consequence memory performance, is impaired by acute and chronic stress [19]. Re-exposure to some stressors could therefore constitute a vulnerability factor for the development of chronic insomnia. The pathophysiology of insomnia has been mainly investigated from the perspective of the hyperarousal model. This approach is based on a long-standing history of clinical observation and empirical findings that patients with insomnia display signs of increased arousal either on a cognitiveemotional, behavioural, autonomous, or central nervous system level. A substantial number of cross-sectional case-control studies have been undertaken to investigate the hypothalamic-pituitary-adrenal axis (with cortisol as the major stress response hormone) and the activity of the autonomic nervous system (heart rate, heart rate 
variability) as indicators for increased arousal levels. Most of the evidence suggests an over activity of these systems in insomnia, supporting the assumption that hyperarousal contributes to insomnia in these patients.

\section{Neurobiology of narcolepsy}

Narcolepsy is a neurological disorder characterized by excessive daytime sleepiness, cataplexy, hypnagonic hallucinations, sleep paralysis, and disturbed nocturnal sleep patterns [20]. There are two distinct subgroups of Narcolepsy: Narcolepsy with cataplexy and Narcolepsy without cataplexy. Family and twin studies have indicated that narcolepsy is a multifactorial disease, triggered by both genetic and environmental factors with the relative risk for first-degree family relatives being 10- to 40 -fold higher than in the general population, and the concordance rate between monozygotic twins being approximately 20\%-30\% [21]. Narcolepsy with cataplexy is generally believed to be associated with the human leukocyte antigen subtype DQB $1 * 0602$ and an intrinsic loss of hypothalamic neurons containing the neuropeptide hypocretin/ orexin with low CSF levels of hypocretin-1, which allows the inappropriate sleep phenomena described above as the classic symptoms narcolepsy [22]. Recent studies have confirmed genetic and epigenetic factors, autoimmune and low CSF hypocretin/ orexin play important roles in the mechanism of narcolepsy [23]. (Table 1).

\section{Neurobiology of circadian rhythm disorders}

This category of disorders includes conditions in which the sleep times are out of alignment. A patient with one of these disorders does not follow the normal sleep times at night. Delayed sleep-wake phase disorder (DSP) is a circadian rhythm disorder with a typical sleep pattern that is "delayed" by two or more hours. This delay occurs when one's internal sleep clock (circadian rhythm) is shifted later at night and later in the morning [24]. Once sleep occurs, the sleep is generally normal. But the delay leads to a pattern of sleep that is later than what is desired or what is considered socially acceptable. This pattern can be a problem when it interferes with work or social demands. A person with DSP is likely to prefer late bedtimes and late wake-up times. When left to his or her own schedule, a person with DSP is likely to have a normal amount and quality of sleep. It simply occurs at a delayed time. One sign of this disorder is difficulty falling asleep until late at night. Another sign is having a hard time getting out of bed in the morning for work or school. These signs can make DSP look like insomnia. Daytime functioning can be severely impaired by DSP. It can lead to excessive sleepiness and fatigue. When able to sleep on their own schedules, people with DSP often stay up until they get tired and then sleep until they awaken late in the morning. In this case, they tend to have no complaint of difficulty falling to sleep or feeling poorly during the day. There is likely to be some genetic component. Some environmental factors may also be involved. A lack of exposure to morning sunlight may make it worse. Too much exposure to bright evening sunlight may also increase symptoms of DSP. A family history of DSP is common in about $40 \%$ of people with the disorder. Drosophila has been a prominent model for research defining the conceptual, functional, and molecular basis of the circadian clock [25]. The circadian clock in Drosophila modulates a broad spectrum of physiological and behavioral processes including locomotor activity, sleep patterns, courtship, learning and memory, feeding behavior, chemosensation, and immune responses [26]. As in mammals, the Drosophila circadian oscillators also coordinate rhythms in peripheral 
organs, such as olfactory and gustatory sensitivity rhythms and the mitotic response of gut stem cells to damage [27].

Konopka and Benzer isolated the first clock gene mutants in 1971 using forward genetics in Drosophila and analysis of the period length of the circadian rhythm in eclosion [28]. Flies with mutations in the gene period $\left(\right.$ per $^{\mathrm{L}}$, per $^{\mathrm{S}}$, and $\left.\mathrm{per}^{01}\right)$ exhibit rhythms in eclosion that are longer, shorter, or arrhythmic, respectively. Identification of per spawned additional genetic screens for components of the circadian clock leading to the discovery of timeless, clock, cycle, doubletime, shaggy, casein kinase 2 subunits and cryptochrome [29]. These studies and subsequent identification of the corresponding genes facilitated research in mammalian systems leading to the discovery of mammalian per and clock genes, the first circadian genes identified and sequenced in mice [30]. The Drosophila central brain circadian system comprises approximately 150 clock neurons organized into a network of oscillators: the small and large ventral lateral neurons which control the morning peak of activity and the lateral dorsal and dorsal neurons that control the evening peak of activity. Circadian rhythms generated by both the Drosophila and mammalian clock are driven by interlocking autoregulatory transcriptional/translational feedback loops along with posttranscriptional regulatory elements that facilitate the rhythmicity of the clock and generate the 24-hour period. As additional information on the molecular mechanisms of the core circadian oscillators in Drosophila and mammals is provided in many excellent reviews [31].

\section{Neurobiology of restless leg syndrome}

Sleep movement disorders include conditions that cause movement during or prior to sleep. These disorders can make it difficult to fall asleep or stay asleep, or to get restful sleep. Most common sleep movement disorder is restless leg syndrome (RLS) and characterized by an urge to move the legs associated with uncomfortable sensations that occur or worsen with rest, are relieved by movements, and are worse in the evening [32]. Motor restlessness is evident when awake and periodic limb movements frequently occur during sleep, resulting in sleep fragmentation and non-restorative sleep. The underlying mechanisms of RLS are largely unknown and even localization of the primary pathology within the nervous system is uncertain. Population and family studies indicate that restless legs syndrome has a strong genetic component and genome-wide association studies have identified genes that confer susceptibility to restless legs syndrome [33]. These studies have successfully identified a number of genetic variants suspected to be involved in restless legs syndrome, including one named BTBD9 [34]. Freeman et al. [35] report how they used the molecular genetic tools available in the model genetic organism Drosophila melanogaster to functionally characterize the fly homologue of BTBD9 (dBTBD9) as a causal factor in restless legs syndrome and loss of the Drosophila homolog CG1826 (dBTBD9) appreciably disrupts sleep with concomitant increases in waking and motor activity. They further show that BTBD9 regulates brain dopamine levels in flies and controls iron homeostasis through the iron regulatory protein- 2 in human cell lines. To our knowledge, this represents the first reverse genetic analysis of a "novel" or heretofore poorly understood gene implicated in an exceedingly common and complex sleep disorder and the development of an RLS animal model that closely recapitulates all disease phenotypes. BTBD9 is a poorly characterized gene that accounts for approximately $50 \%$ of the population attributable risk for restless legs syndrome and is therefore an ideal candidate for functional analysis using Drosophila genetics. Freeman et al. identified the fly homologue of BTBD9 and created two null mutations in the gene [35]. When they examined sleep in these mutants, they found that both mutant alleles caused severely fragmented nighttime sleep and an increase in the amount of waking after sleep onset, as is seen in human patients with restless legs syndrome. Because sleep fragmentation is only one component of restless legs syndrome, the authors needed to evaluate 
aspects of movement that have face-validity with symptoms seen in human patients with restless legs syndrome. In humans, the diagnosis of restless legs syndrome is facilitated by the 'suggested immobilization test', in which periodic limb movements and leg discomfort are worsened when subjects are asked to sit in bed with their legs outstretched for an hour [36]. Intriguingly, when mutants in dBTBD9 were confined to a small space, to simulate the suggested immobilization test, they showed significantly more locomoter behavior than controls. Moreover, when locomotor behavior was precisely quantified in an independent assay, the mutants were found to spend more time in longer uninterrupted bouts of walking than controls. Together, these data indicate that dBTB9 mutant flies recapitulate key behavioral aspects of restless legs syndrome, including sleep fragmentation and motor restlessness.

Recently, Ferre et al. [37], reported that alterations in the dopaminergic system seem to be involved in RLS, while alterations in glutamatergic neurotransmission, a presynaptic hyperglutamatergic state, seem to be involved in hyperarousal and also RLS. Brain iron deficiency is well-recognized as a main initial pathophysiological mechanism of RLS. Brain iron deficiency in rodents have provided a pathogenetic model of RLS that recapitulates the biochemical alterations of the dopaminergic system of RLS, although without PLMS-like motor abnormalities. In addition, Ferre et al have showed that brain iron deficiency in rodents is associated with changes in adenosinergic transmission, with downregulation of adenosine $A_{1}$ receptors (A1R) as the most sensitive biochemical finding. It is likely that A1R downregulation leads to hypersensitive striatal glutamatergic terminals and facilitation of striatal dopamine release. Hypersensitivity of striatal glutamatergic terminals was demonstrated by an optogenetic-microdialysis approach in the animal model, indicating that it could represent a main pathogenetic factor that leads to PLMS in RLS.

\section{Conclusion}

Neurobiological mechanisms of sleep disorders are complex and poorly understood. Most of the pathological process are multifactorial and variable. Recent progress in molecular genetics has enable researchers to use genetic approach to understand the pathophysiology of these disorders. Indeed, studies in Drosophila have yielded new insights into basic questions regarding sleep function and regulation. More recently, powerful genetic approaches in the fly have been applied toward studying primary human sleep disorders and other disorders associated with dysregulated sleep. Remarkable progress has been made in the area of genetics and immunology related to common sleep disorders. These progress will have a significant impact on the clinical practice of sleep medicine in the future. As we have reviewed, the new information regarding the genetic and molecular mechanism underlying common sleep disorders, such as insomnia, circadian rhythm disorders, narcolepsy, restless legs syndrome will likely lead to improved diagnostic tests and new individualized treatment.

\section{References}

1. Jones BE. From waking to sleeping: neuronal and chemical substrates. Trends in Pharmacol Sci. 2005; 26: 578-586. Ref.: https://tinyurl.com/yc9pwoy4

2. Tolaymat A, Liu Z. Sleep disorders in childhood neurological diseases. Children (Basel). 2017; 4: 8498. Ref.: https://tinyurl.com/y9rvrpd7

3. Brown RE, Basheer R, McKenna JT, Strecker RE, McCarley RW. Control of sleep and wakefulness. Physiol Rev. 2012: 92: 1087-1187. Ref.: https://tinyurl.com/yb6x7cu9

4. Schwartz MD, Kliduff TS. The neurobiology of sleep and wakefulness. Psychiatr Clin North Am. 2015: 38: 615-644. Ref.: https://tinyurl.com/yd2kzpq3

5. Hendricks JC, Kirk D, Pancheri K, Miller MS, Pack Al. Modafinil maintains waking in the fruit fly drosophila melanogaster. Sleep. 200; 26: 139-146. Ref.: https://tinyurl.com/yakzxkqd

6. Wager-Smith K, Kay SA. Circadian rhythm genetics: From flies to mice to humans. Nature Genetics 2000; 26: 23-27. Ref.: https://tinyurl.com/ybbao8hq 
7. Herber R, Hill SL, Holladay C, Biesiadecki M, Tononi G, et al. Sleep homeostasis in Drosophila melangaster. Sleep. 2004; 27: 628-639. Ref.: https://tinyurl.com/y7zaeruy

8. Cirelli C. The genetic and molecular regulation of sleep: From fruit flies to humans. Nat Rev Neurosci. 2009; 10: 549-560. Ref.: https://tinyurl.com/yalawocu

9. Lamberg L. Manual updates sleep disorder diagnoses. Psychiatric news. 2014.

10. Roth T. Insomnia: Definition, prevalence, etiology, and consequences. J Clin Sleep Med. 2007; 3: 7-10. Ref.: https://tinyurl.com/ybmwegft

11. Feige B, Al-Shajlawi A, Nisseb C, Voderholzer U, Hornyak M, et al. Does REM sleep contribute to subjective wake time in primary insomnia? A comparison of polysomnographic and subjective sleep in 100 patients. J Sleep Res. 2008; 7: 180-190. Ref.: https://tinyurl.com/y8by6557

12. Wing YK, Zhang J, Lam SO, Li SX, Lai KY, et al. Familial aggregation and heritability of insomnia in a community-based study. Sleep Med. 2012; 13: 985-990. Ref.: https://tinyurl.com/yau9eqlt

13. Barclay NL Eley TC, Buysse DJ, Archer SN, Gregory AM. Diurnal preference and sleep quality: same genes? A study of young adult twins. Chronobiol Int. 2010; 27: 278-296. Ref.: https://tinyurl.com/ycnvhjpc

14. Josephs KA, Silber MH, Fealey RD, Nippoldt TB, Auge RG, et al. Neurophysiologic studies in Morvan syndrome. J Clin Neurophysiol. 2004; 21: 440-445. Ref.: https://tinyurl.com/y75qzz5s

15. Serretti A, Benedetti F, Mandelli L, Lorenzi C, Pirovano A, et al. Genetic dissection of psychopathological symptoms: insomnia in mood disorders and CLOCK gene polymorphism. Am J Med Genet B Neuropsychiatr Genet, 2003; 121: 35-38. Ref.: https://tinyurl.com/yanatexl

16. Buhr A, Bianchi MT, Baur R, Courtet $P$, Pignay $V$, et al. Functional characterization of the new human $\mathrm{GABA}(\mathrm{A})$ receptor mutation beta3(R192H). Hum Genet. 2002; 111: 154-160. Ref.: https://tinyurl.com/yauwaq5q

17. Deuschle $M$, Schredl $M$, Schilling $C$, Wust $S$, Frank J, et al. Association between a serotonin transporter length polymorphism and primary insomnia. Sleep. 2010; 33: 343-347. Ref.: https://tinyurl.com/yaq6ytnc

18. Barclay NL, Gregory AM. Quantitative genetic research on sleep: a review of normal sleep, sleep disturbances and associated emotional, behavioral and health-related difficulties. Sleep Med Rev. 2013; 17: 29-40. Ref.: https://tinyurl.com/y9p54kft

19. Riemann D, Spiegelhalder K, Feige B, Voderholzer U, Berger $M$, et al. The hyperarousal model of insomnia: a review of the concept and its evidence. Sleep Med Rev. 2010; 14: 19-31. Ref.: https://tinyurl.com/y8b7nbd2

20. Longstreth WT, Koepsell TD, Ton TG, Hendrickson AF, Van Bell B. The epidemiology of narcolepsy. Sleep. 2007; 30: 13-26. Ref.: https://tinyurl.com/yb94qq9m

21. Khatami R, Maret S, Werth E, Retey J, Schmid D, et al. Monozygotic twins concordant for narcolepsycataplexy without any detectable abnormality in the hypocretin (orexin) pathway. Lancet. 2004; 363: 1199-1200. Ref.: https://tinyurl.com/ydgp3r9e

22. Mignot E. Genetic and familial aspects of narcolepsy. Neurology. 1998; 50: 16-22. Ref.: https://tinyurl.com/y9vzmget

23. Liu Z, Tolarmat A, Sreenivas KA. Neurobiology of narcolepsy. J Sleep Med \& disorders. 2018; 5 : 1093-1098.

24. Konopka R, Pittendrigh C, Orr D. Reciprocal behavior associated with altered homeostasis and photosensitivity of Drosophila clock mutants. J Neurogenet. 1989; 6: 1-10. Ref.: https://tinyurl.com/yakfvdms

25. Zhang Y, Ling Y, Yuan C, Dubruille R, Emery P. A role for Drosophila ATX2 in activation of PER translation and circadian behavior. Science. 2013; 340: 879-882. Ref.: https://tinyurl.com/ydz2tafk

26. Chatterjee A. Hardin P. E. Time to taste: circadian clock function in the Drosophila gustatory system. Fly. 2010; 4: 283-287. Ref.: https://tinyurl.com/y9f7pzu3

27. Karpowicz $P$, Zhang $Y$, Hogenesch JB, Emery P, Perrimon N. The circadian clock gates the intestinal stem cell regenerative state. Cell Reports. 2013; 4: 996-1004. Ref.: https://tinyurl.com/ycv4v3k6

28. Konopka RJ. Benzer S. Clock mutants of Drosophila melanogaster. Proceedings of the National Academy of Sciences of the United States of America. 1971; 68: 2112-2116. Ref.: https://tinyurl.com/ybgkrkev

29. Fan JY, Agyekum B, Venkatesan Y, Hall DR, Keightley A, et al. Noncanonical FK506-binding protein BDBT binds DBT to enhance its circadian function and forms foci at night. Neuron. 2013; 80: 984 996. Ref.: https://tinyurl.com/y94vj363 
30. Tei $H$, Okamura $H$, Shigeyoshi $Y$, Fukuhara $C$, Ozawa $R$, et al. Circadian oscillation of a mammalian homologue of the Drosophila period gene. Nature. 1997; 389: 512-516. Ref.: https://tinyurl.com/yck54vy2

31. Dubowy C. Sehgal A. Circadian rhythms and sleep in Drosophila melanogaster. Genetics. 2017; 205: 1373-1397. Ref.: https://tinyurl.com/yaax9ztv

32. Yeh $P$, Walters AS, Tsuang JW. A comprehensive overview on its epidemiology, risk factors and treatment. Subst Use Misuse. 2011; 47: 9-10.

33. Sehgal A. Mignot E. Genetics of sleep and sleep disorders. Cell. 2011; 146: 194-207. Ref.: https://tinyurl.com/y7guppqa

34. Yang Q, Li L, Chen Q, Foldvary-Schaefer N, Ondo WG, et al. Association studies of variants in MEIS1, BTBD9, and MAP2K5/SKOR1 with restless legs syndrome in a US population. Sleep Med. 2011; 12: 800-804. Ref.: https://tinyurl.com/ycq99qdc

35. Freeman A, Pranski E, Miller RD, Radmard S, Bernhard D, et al. Strong fragmentation and motor restless in Drosohilia model of Restless legs syndrome. Curr Biol, 2012; 22: 1142-1148. Ref.: https://tinyurl.com/y8tx33r5

36. Michaud M, Lavigne G, Dsaulels A, Poirier G, Montplaisir J. Effects of immobility on sensory and motor symptoms of restless legs syndromee. J Mov Dis Soc. 2002; 17: 112-115. Ref.: https://tinyurl.com/ycjmzm95

37. Ferre S, Quiroz C, Guitart X, Rea W, Seyedian A, et al. Pivotal role of adenosine neurotransmittion in restless legs syndrome. Front Neurosci. 2018; 8: 722-736. Ref.: https://tinyurl.com/yaep4ymv 